\title{
Maternal mRNA deadenylation is defective in in vitro matured mouse and human
}

\section{oocytes}

Yusheng Liu ${ }^{1,5}$, Wenrong $\mathrm{Tao}^{3,5}$, Yiwei Zhang ${ }^{1,4}, \mathrm{Hu} \mathrm{Nie}^{1,2}$, Zhenzhen $\mathrm{Hou}^{3}$, Jingye Zhang $^{3}$, Zhen Yang ${ }^{3}$, Jiaqiang Wang ${ }^{4}$, Falong $\mathrm{Lu}^{1,2} \bowtie$ and Keliang $\mathrm{Wu}^{3 凶}$

${ }^{1}$ State Key Laboratory of Molecular Developmental Biology, Institute of Genetics and Developmental Biology, Innovative Academy of Seed Design, Chinese Academy of Sciences, Beijing 100101, China.

${ }^{2}$ University of Chinese Academy of Sciences, Beijing 100049, China.

${ }^{3}$ Center for Reproductive Medicine, Shandong University, The Key laboratory of Reproductive Endocrinology, Ministry of Education, Shandong University, Jinan 250012, China.

${ }^{4}$ College of Life Science, Northeast Agricultural University, Harbin 150030, China.

${ }^{5}$ These authors contributed equally: Yusheng Liu, Wenrong Tao.

凶e-mail: liuys1126@foxmail.com; wangjiaqiang@neau.edu.cn; fllu@genetics.ac.cn; wukeliang_527@163.com. 


\begin{abstract}
Oocyte in vitro maturation is a technique of assisted reproductive technology that was first introduced in patients with polycystic ovarian syndrome and is now used in most fertility clinics. Thousands of genes show abnormally high expression in in vitro maturated metaphase II (in vitro MII) oocytes compared with in vivo maturated metaphase II (in vivo MII) oocytes in bovines, mice, and humans ${ }^{1-3}$. However, the underlying mechanisms of this abnormal expression are still poorly understood. In this study, we use PAIso-seq1 to reveal a transcriptome-wide expression profile of full-length transcripts containing entire poly(A) tails in in vivo and in vitro matured mouse and human oocytes. Our results indicate that more genes are up-regulated than down-regulated in in vitro MII oocytes in both mice and humans. Furthermore, we demonstrate that the observed increase in maternal mRNA abundance is caused by impaired deadenylation in in vitro MII oocytes in both mice and humans. We also found that the cytoplasmic polyadenylation of dormant Btg4 and Cnot7 mRNAs, which encode key components of deadenylation machinery, is impaired in in vitro MII oocytes in mice and humans respectively, likely contributing to reduced translation and impaired global maternal mRNA deadenylation. Our findings highlight that impaired maternal mRNA deadenylation is a definite molecular defect in in vitro MII oocytes in both mice and humans. The findings here offer a new criterion for evaluating the quality of in vitro MII oocytes and a potential direction for improving in vitro maturation by fixing the dysregulated maternal mRNA deadenylation.
\end{abstract}




\section{Introduction}

Female infertility is becoming an exacerbating reproductive problem, for which the assisted reproductive technology (ART) is an effective treatment ${ }^{4}$. In vitro maturated metaphase II (in vitro MII) oocytes were first introduced as an assisted reproductive technology for patients with polycystic ovarian syndrome (PCOS) ${ }^{5}$ and patients with severe ovarian hyperstimulation syndrome (OHSS) during previous in vitro fertilization (IVF) treatments ${ }^{6}$. Currently, in vitro MII oocytes can be adapted as an option in almost all areas of fertility clinics, including PCO-like ovaries, resistant ovary syndrome, previous failed IVF attempts, and oocyte maturation problems ${ }^{7}$. Additionally, in vitro MII oocytes can be useful for preserving fertility in situations such as emergency oocyte retrieval due to malignancies ${ }^{8}$. The first baby from donor in vitro MII oocytes was born in $1991^{9}$, and the first baby from the mother's own in vitro MII oocytes was born in $1994^{10}$. As such, in recent years, in vitro MII oocytes have gained increasing attention for their feasibility, safety, reproducibility, cost-effectiveness, and lack of OHSS risk ${ }^{10,11}$.

Previous studies have demonstrated that embryos derived from in vitro MII oocytes have a lower success rates of preimplantation development, pregnancy, and birth than those derived from mature in vivo MII (in vivo MII) oocytes ${ }^{12}$. The nuclear and cytoplasmic maturation in in vitro MII oocytes determines the performance of their oocytes, the quality of the embryo, and clinical outcomes ${ }^{7,13}$. The nuclear maturation can be easily evaluated with a microscope based on the first polar body extrusion. The cytoplasmic maturation defects during the in vitro maturation process include altered spindle positioning, mitochondrial membrane potential, the number of endoplasmic reticulum clusters, and the cortical actin cytoskeleton thickness in in vitro MII oocytes compared with in vivo MII oocytes $^{14,15}$. Global gene expression profiling using human whole-genome arrays provides compelling evidence for the relative developmental incompetence of in vitro MII oocytes ${ }^{1}$, demonstrating that over 2,000 genes were expressed at 2-fold or greater levels in in vitro MII oocytes compared with in vivo MII oocytes. In vitro MII oocytes in mice and bovines 
were also demonstrated elevated expression patterns ${ }^{2,3}$. Nonetheless, the mechanisms underlying cytoplasmic maturation defects and upregulated gene expression profiles in in vitro MII oocytes remain largely unknown.

As transcription is silent, post-transcriptional regulation of maternal mRNA plays a dominant role in oocyte maturation, particularly deadenylation-dependent maternal mRNA decay. The CCR4-NOT deadenylase and its adaptor Btg4 are critical regulators for this process $^{16-18}$. In addition, Cnot7 and Cnot6l, which encode catalytic subunit of the CCR4NOT deadenylase, and Btg4 are dormant maternal mRNAs that needs to be translationally activated through cytoplasmic polyadenylation during mouse oocyte maturation ${ }^{16,17}$. We demonstrated that maternal mRNAs are subjected to deadenylation-dependent decay during oocyte maturation in mice, rats, pigs, and humans ${ }^{19-23}$. Therefore, we hypothesize that the global gene upregulation observed in the in vitro MII oocytes is caused by impaired global deadenylation. To test this hypothesis, we used the PAIso-seq1 method, which is capable of measuring poly(A) tail inclusive full-length transcriptome from a single oocyte ${ }^{24}$, to measure the transcriptome-wide poly(A) tails in in vivo and in vivo MII oocytes in both mice and humans. By comparing the transcriptome-wide poly(A) tail length distribution of maternal mRNA in in vitro MII and in vivo MII oocytes, we found that the maternal mRNA decay mediated by poly(A) tail deadenylation was impaired in in vitro MII oocytes in both mice and humans. Furthermore, we found that cytoplasmic polyadenylation is critical for the translational activation of Btg4 and Cnot7 mRNAs, both of which encode factors critical for global maternal mRNA deadenylation and were impaired in in vitro MII oocytes in mice and humans. Therefore, our findings reveal that impaired maternal mRNA deadenylation is a critical defect in in vitro MII oocytes of both mice and humans, and provide the groundwork for improving the quality of in vitro MII oocytes.

\section{Results}

Abnormal maternal gene expression in in vitro MII oocytes in mice and humans 
The deadenylation and cytoplasmic polyadenylation of the poly(A) tail of maternal mRNA both play essential roles during oocyte maturation ${ }^{25,26}$. Therefore, we performed PAIsoseq1 to analyze the poly(A) tail inclusive transcriptome in in vitro and in vivo MII oocytes as well as the germinal vesicle (GV) oocytes in both mice and humans (Fig. 1a, f). We found that the overall transcriptional level of individual genes significantly decreased during maturation in both mice and humans (Fig. 1b, g). This is consistent with the wellknown global reduction of maternal mRNA during mammalian oocyte maturation, as measured by RNA-seq or microarray ${ }^{1,16,17}$. The transcriptional level of individual genes was significantly higher in in vitro than in in vivo MII oocytes in mice and humans (Fig. $1 \mathrm{~b}, \mathrm{~g})$, indicating that global maternal mRNA decay is impaired in in vitro MII oocytes. We found that 4,789 genes increased, while only 343 genes decreased in in vitro compared to in vivo MII oocytes in mice (Fig. 1c), and 3,072 genes increased while only 1,352 genes decreased in in vitro compared to in vivo MII oocytes in humans (Fig. 1h). The up-regulated genes enrich in cellular metabolic process, organelle organization, cell cycle, as well as DNA repair in both mice and humans (Fig. 1d, i). This indicates the presence of conserved defects in in vitro MII oocytes between mice and humans. Bmp15 is known to inhibit follicle maturation and is downregulated during this process ${ }^{27}$; we found that it is upregulated in in vitro compared to in vivo MII oocytes in both mice and humans (Fig. 1e, j). Our findings are consistent with previous global gene expression profiling comparing in vitro and in vivo MII oocytes in humans and mice ${ }^{1,2}$ and were similar when comparing pluripotent stem cells (PSCs)-derived MII oocytes and in vivo MII oocytes of mouse ${ }^{28}$. These results indicate that the impaired maternal mRNA decay is one conserved defect in in vitro MII oocytes in both mice and humans.

\section{Impaired maternal mRNA deadenylation in in vitro MII oocytes of mouse and human}

In eukaryotes, most mRNA decay is initiated by poly(A) tail deadenylation, including maternal mRNA decay during mammalian oocyte maturation ${ }^{29}$. It has been reported that 
deadenylation inhibition via Btg4 depletion in mouse oocytes ${ }^{16,17}$ or Btg4 mutation in human oocytes ${ }^{18}$ impedes maternal mRNA decay. Therefore, we explored whether the impaired maternal mRNA decay seen in in vitro MII oocytes is due to defective RNA deadenylation. To test this, we analyzed the transcriptome-wide poly(A) tail length distribution in in vitro and in vivo MII oocytes and found a global accumulation of transcripts with 20-100 nt poly(A) tails in in vitro MII oocytes in mice and humans (Fig. 2a, e). This indicates impaired maternal mRNA deadenylation, which is typically more severe in transcripts from genes that are upregulated in in vitro MII oocytes (Figs 1c, h, 2b, f), while transcripts from genes that are downregulated in in vitro MII oocytes showed an opposite pattern (Figs 1c, h, 2c, g). For example, Bmp 15 transcripts with 20-100 nt poly(A) tails were significantly accumulated in in vitro MII oocytes of both mice and humans (Fig. $2 \mathrm{~d}, \mathrm{~h})$. These results demonstrate that the deadenylation of maternal mRNA is impaired in in vitro MII oocytes, which is conserved in both mice and humans.

We report that non-A residues can be incorporated into maternal mRNA poly(A) tails through cytoplasmic polyadenylation followed by deadenylation during oocyte maturation, which is conversed in mice, rats, pigs, and humans ${ }^{19-23}$. When the deadenylation is impaired, the $\mathrm{N}$ (length between the end of 3' UTR and the longest consecutive U, C, or G residues in poly (A) tails, Fig. 3a, c) is expected to be longer. This has been validated that the knockdown of Btg4 in human zygotes and mouse MII oocytes results in longer $\mathrm{N}^{20,21}$. Therefore, we hypothesized that $\mathrm{N}$ should be longer in in vitro MII oocytes in which maternal mRNA deadenylation was impaired. As a result, we found that the $\mathrm{N}$ was longer for poly(A) tails with $U$ residues in in vitro than in in vivo MII oocytes in both mice and humans (Fig. 3b, d). We also noticed that the ratio of poly(A) tails with $\mathrm{C}$ or $\mathrm{G}$ residues was lower in in vitro than in in vivo MII oocytes in mice and humans (Fig. 3b, d), reflecting lower incorporation of $\mathrm{C}$ and $\mathrm{G}$ residues (Extended Data Fig. 1). This suggests different mechanisms in $\mathrm{U}$ incorporation and $\mathrm{C} / \mathrm{G}$ incorporation during oocyte maturation.

Altogether, these data demonstrate that impaired maternal mRNA decay is caused by 
impaired deadenylation in in vitro MII oocytes in both mice and humans.

\section{Impaired cytoplasmic polyadenylation of Btg4 and Cnot7 in in vitro MII oocytes}

Recent studies have demonstrated that CCR4-NOT deadenylase and its adaptor BTG4 are critical for global maternal mRNA deadenylation during mouse and human OET ${ }^{16-18,21,22}$. Therefore, we examined whether the impaired deadenylation in in vitro MII oocytes was caused by problems that occurred with CCR4-NOT deadenylase and its adaptor BTG4. The poly(A) tails of Btg4 and Cnot7 mRNA are short at the GV stage, and their translation is not initiated until cytoplasmic polyadenylation during oocyte maturation ${ }^{16,17,30}$. Therefore, we analyzed the transcript level and the poly(A) tail length of genes encoding components of the CCR4-NOT deadenylase and its adaptor BTG4 in in vitro MII oocytes of mice and humans.

At the transcript level, we found no or slight changes in the expression of these genes between in vitro and in vivo MII oocytes in mice and humans, except that Cnot 8 decreased dramatically in in vitro MII oocytes in humans (Fig. 4a, d). At the poly(A) tail length level, we observed a dramatic increase in poly(A) tail length for these genes during in vivo oocyte maturation in mice and humans (Fig. 4b, e), reflecting the cytoplasmic polyadenylation of these genes. However, we found that the cytoplasmic polyadenylation of Btg4 in in vitro MII oocytes in mice and CNOT7 in in vitro MII oocytes in humans was heavily impaired (Fig. 4b, e), suggesting that these factors cannot be translated at a normal level due to defective cytoplasmic polyadenylation.

The poly(A) tail length of mRNA is known to be positively associated with translational efficiency (TE) in mice GV to MI stage oocytes ${ }^{22}$. This positive association between poly(A) tail length and TE of mRNA has been suggested in mammalian MII oocytes but has never been examined transcriptome-widely. Therefore, we analyzed the relationship between our poly(A) tail length data and TE data from published polysome-seq in mouse MII oocytes ${ }^{31}$. We found that genes with longer poly(A) tails showed significantly higher 
TE in MII oocytes (Fig. 4c). For example, Btg4, Tcl1b4, and Wee2 with poly(A) tail lengths around $140 \mathrm{nt}$ showed much higher TE than $S u z 12, \operatorname{Tg} f b 2$, and $\operatorname{Atg} 5$ with poly(A) tail lengths around $50 \mathrm{nt}$ (Fig. 4c). Altogether these results indicate that impaired deadenylation in in vitro MII oocytes is associated with the impaired cytoplasmic polyadenylation of mRNAs encoding CCR4-NOT deadenylase and its adaptor BTG4.

\section{Discussion}

In vitro MII oocytes initially gained attention in ART for patients with $\operatorname{PCOS}^{5}$. More recently, it has been used in patients with repeated IVF failures ${ }^{10}$, resistant ovary syndrome, oocyte maturation problems $^{7}$, and hormone-sensitive tumors ${ }^{8}$. Combining in vitro maturation and cryopreservation provides new opportunities for women to postpone motherhood $^{7}$. The reduced ability of in vitro MII oocytes to support embryo development has been reported, however, the molecular mechanism underlying its defect is still unclear. In this study, we revealed that the maternal mRNA decay mediated by deadenylation is defective in in vitro MII oocytes in both mice and humans, which is associated with the defective cytoplasmic polyadenylation of mRNAs encoding CCR4-NOT deadenylase and its adaptor BTG4 (Fig. 5). These defects could potentially be used also as biomarkers in evaluating oocyte quality following the in vitro maturation procedure.

In addition to impaired maternal mRNA decay, we revealed that the incorporation of non-A residues into poly(A) tails were also impaired during oocyte maturation, showing lower ratio of poly(A) tails with non-A residues, especially the $\mathrm{C}$ and $\mathrm{G}$ residues, in in vitro than in in vivo MII oocytes in both mice and humans (Extended Data Fig. 1). This was associated with the impaired cytoplasmic polyadenylation of Tent $4 a / b$ in in vitro MII oocytes which encode enzymes adding $\mathrm{C}$ and $\mathrm{G}$ residues to poly $(\mathrm{A})$ tails ${ }^{32}$, although the expression levels of Tent4a/b were higher in in vitro than in in vivo MII oocytes of mouse and human (Extended Data Fig. 2). The mechanism behind how the cytoplasmic polyadenylation of Btg4, Cnot7, and Tent4a/b is impaired in in vitro MII oocytes warrants 
further exploration in the future.

BTG4-mediated maternal mRNA decay is essential for successful OET in mice and humans, since Btg4-null oocytes were infertile due to developmental arrest at the cleavage stage $^{16-18}$. In this study, we demonstrated that maternal mRNA deadenylation is impaired in mouse and human in vitro MII oocytes, which is associated with decreased developmental potential for supporting embryonic development. Improving the activity of CCR4-NOT deadenylase and its adaptor BTG4 is likely to help solving this problem, and we expect that these approaches can one day promote the cytoplasmic maturation of oocytes under in vitro conditions. This will ultimately promote the future use of in vitro MII oocytes in human ART clinics.

Oocytes have recently been created in vitro from mouse pluripotent stem cells (PSCs), which can give rise to fertile and healthy offspring ${ }^{28,33-36}$. These groundbreaking results prove that it is possible to reproduce mice in the absence of natural oocytes. Using these methods in humans could provide hope for patients with infertility caused by oocyte abnormalities. Additionally, this procedure can contribute to regenerative medicine by providing oocytes for therapeutic cloning. Notably, PSC-derived MII oocytes also showed a higher level of gene expression of maternal mRNA than in vivo MII oocytes ${ }^{28}$. Therefore, our findings can also be used to evaluate the quality of and improve PSC-derived oocytes, a subject that warrants further study. 


\section{Materials and Methods}

\section{Human oocytes}

The use of human gametes for this research follows the Human Biomedical Research Ethics Guidelines (set by National Health Commission of the People's Republic of China on 1 December 2016), the 2016 Guidelines for Stem Cell Research and Clinical Translation (issued by the International Society for Stem Cell Research, ISSCR) and the Human Embryonic Stem Cell Research Ethics Guidelines (set by China National Center for Biotechnology Development on 24 December 2003). All the human related experiments in this study are in compliance with these relevant ethical regulations. The aim and protocols of this study has been reviewed and approved by the Institutional Review Board of Reproductive Medicine, Shandong University.

The donor women are 25-38 years old with tubal-factor infertility and their partners have healthy semen. Written informed consent was obtained from all oocyte and sperm donors. Oocytes donated from patients taking in-vitro fertilization treatments. Immature human GV oocytes without cumulus cells were matured in in vitro maturation (IVM) medium at $37{ }^{\circ} \mathrm{C}$ in an atmosphere with $5 \% \mathrm{CO}_{2}$ for 24 hours. The IVM medium consists of M199 medium (GIBCO, 11-150-059) with 20\% Systemic Serum Substitute (Irvine Scientific, 99193) and $75 \mathrm{mIU} / \mathrm{mL}$ of recombinant follicle stimulating hormone (Merck Serono). The oocytes were randomly assigned to experimental groups. Single oocytes were used for PAIso-seq1 analysis with 3 - 4 replicates for each stage.

\section{Mouse oocytes}

CD1 (ICR) Mice were purchased from Beijing Vital River Laboratory Animal Technology Co., Ltd. and maintained according to the guidelines of the Animal Care and Use Committee of the Institute of Genetics and Development Biology, Chinese Academy of Sciences. GV oocytes were isolated from ovaries after injection with $10 \mathrm{U}$ of pregnant mare serum gonadotropin (PMSG) (Prospec). GV oocytes without cumulus cells were in vitro cultured in M16 medium for 14 hours to collect in vitro matured MII oocytes. To obtain 
MII oocytes, the mice were injected with $10 \mathrm{U}$ of PMSG and $10 \mathrm{U}$ of human chorionic gonadotropin (hCG) (Prospec) at 46- to 48-hour intervals. MII oocytes were isolated from the oviduct, without mating, 14 hours after hCG injection.

\section{PAIso-seq1 library construction}

The PAIso-seq1 libraries for human single oocytes were constructed following the singlecell PAIso-seq1 protocol according to previously described methods ${ }^{24}$. The PAIso-seq1 libraries for mouse oocytes were constructed with total RNA prepared from mouse oocytes using TRIzol reagent (Life Technologies). The libraries sequenced using PacBio Sequel II instruments at Annoroad.

\section{Data availability}

The ccs data in bam format from PAIso-seq1 experiments will be available at Genome Sequence Archive hosted by National Genomic Data Center. This study includes analysis of the following published data: Sha et al. (Gene Expression Omnibus database (GEO) accession no. GSE118564). Custom scripts used for data analysis will be available upon request.

\section{Acknowledgements}

This work was supported by the National Key Research and Development Program of China (2018YFC1004000), the Strategic Priority Research Program of the Chinese Academy of Sciences (XDA24020203), National Natural Science Foundation of China (31970588, 32170606, 81871168), Natural Science Foundation of Heilongjiang province (YQ2020C003), the China Postdoctoral Science Foundation (2020M670516, 2020T130687), the State Key Laboratory of Molecular Developmental Biology, and the Fundamental Research Funds of Shandong University.

\section{Author Contributions}

Yusheng Liu, Jiaqiang Wang and Falong Lu conceived the project and designed the study. Yusheng Liu, Yiwei Zhang, Hu Nie, Jiaqiang Wang and Falong Lu analyzed the sequencing data. Wenrong Tao, Zhenzhen Hou, Jingye Zhang, Zhen Yang and Keliang Wu collected 
human oocytes. Yusheng Liu and Jiaqiang Wang organized all figures. Yusheng Liu, Jiaqiang Wang and Falong Lu supervised the project. Yusheng Liu, Jiaqiang Wang and Falong Lu wrote the manuscript with the input from the other authors.

\section{Competing Interests statement}

The authors declare no competing interests.

\section{Reference}

1 Jones, G. M. et al. Gene expression profiling of human oocytes following in vivo or in vitro maturation. Hum Reprod 23, 1138-1144, doi:10.1093/humrep/den085 (2008).

2 Zhang, H. L. et al. Increased Environment-Related Metabolism and Genetic Expression in the In Vitro Matured Mouse Oocytes by Transcriptome Analysis. Front Cell Dev Biol 9, 642010, doi:10.3389/fcell.2021.642010 (2021).

3 Katz-Jaffe, M. G., McCallie, B. R., Preis, K. A., Filipovits, J. \& Gardner, D. K. Transcriptome analysis of in vivo and in vitro matured bovine MII oocytes. Theriogenology 71, 939-946, doi:10.1016/j.theriogenology.2008.10.024 (2009).

4 Gulimiheranmu, M., Wang, X. \& Zhou, J. Advances in Female Germ Cell Induction from Pluripotent Stem Cells. Stem cells international 2021, 8849230, doi:10.1155/2021/8849230 (2021).

Delvigne, A. \& Rozenberg, S. Review of clinical course and treatment of ovarian hyperstimulation syndrome (OHSS). Hum Reprod Update 9, 77-96, doi:10.1093/humupd/dmg005 (2003).

Ata, B., Yakin, K., Balaban, B. \& Urman, B. Embryo implantation rates in natural and stimulated assisted reproduction treatment cycles in poor responders. Reprod Biomed Online 17, 207-212, doi:10.1016/s1472-6483(10)60196-4 (2008).

Hatirnaz, S. et al. Oocyte in vitro maturation: A sytematic review. Turkish journal of obstetrics and gynecology 15, 112-125, doi:10.4274/tjod.23911 (2018).

Lindenberg, S. New approach in patients with polycystic ovaries, lessons for everyone. Fertil Steril 99, 1170-1172, doi:10.1016/j.fertnstert.2013.02.007 (2013).

9 Cha, K. Y. et al. Pregnancy after in vitro fertilization of human follicular oocytes collected from nonstimulated cycles, their culture in vitro and their transfer in a donor oocyte program. Fertil Steril 55, 109-113, doi:10.1016/s0015-0282(16)54068-0 (1991).

Trounson, A., Wood, C. \& Kausche, A. In vitro maturation and the fertilization and developmental competence of oocytes recovered from untreated polycystic ovarian patients. Fertil Steril 62, $353-$ 362, doi:10.1016/s0015-0282(16)56891-5 (1994).

11 Soderstrom-Anttila, V., Makinen, S., Tuuri, T. \& Suikkari, A. M. Favourable pregnancy results with insemination of in vitro matured oocytes from unstimulated patients. Hum Reprod 20, 1534-1540, doi:10.1093/humrep/deh768 (2005).

12 Guzman, L. et al. Developmental capacity of in vitro-matured human oocytes retrieved from polycystic ovary syndrome ovaries containing no follicles larger than $6 \mathrm{~mm}$. Fertil Steril 98, 503-507 e501-502, doi:10.1016/j.fertnstert.2012.01.114 (2012). 
Mohsenzadeh, M., Salehi-Abargouei, A., Tabibnejad, N., Karimi-Zarchi, M. \& Khalili, M. A. Effect of vitrification on human oocyte maturation rate during in vitro maturation procedure: A systematic review and meta-analysis. Cryobiology 83, 84-89, doi:10.1016/j.cryobiol.2018.05.003 (2018). Moon, J. H. et al. Spindle positions and their distributions in in vivo and in vitro matured mouse oocytes. Hum Reprod 20, 2207-2210, doi:10.1093/humrep/dei044 (2005). Ferrer-Vaquer, A., Barragan, M., Rodriguez, A. \& Vassena, R. Altered cytoplasmic maturation in rescued in vitro matured oocytes. Hum Reprod 34, 1095-1105, doi:10.1093/humrep/dez052 (2019). Liu, Y. et al. BTG4 is a key regulator for maternal mRNA clearance during mouse early embryogenesis. J Mol Cell Biol 8, 366-368, doi:10.1093/jmcb/mjw023 (2016).

$\mathrm{Yu}, \mathrm{C}$. et al. BTG4 is a meiotic cell cycle-coupled maternal-zygotic-transition licensing factor in oocytes. Nat Struct Mol Biol 23, 387-394, doi:10.1038/nsmb.3204 (2016).

Zheng, W. et al. Homozygous Mutations in BTG4 Cause Zygotic Cleavage Failure and Female Infertility. Am J Hum Genet 107, 24-33, doi:10.1016/j.ajhg.2020.05.010 (2020).

Liu, Y. et al. Re-polyadenylation occurs predominantly on maternal mRNA degradation intermediates during mammalian oocyte-to-embryo transition. bioRxiv Processing (2021).

Liu, Y. et al. Abundant non-A residues in the poly(A) tail orchestrate the mouse oocyte-to-embryo transition. bioRxiv Processing (2021).

21 Liu, Y. et al. Dynamics of poly(A) tail length and non-A residues during the human oocyte-to-embryo transition. bioRxiv Processing (2021).

22 Liu, Y. et al. Poly(A) tail length is a major regulator of maternal gene expression during the mammalian oocyte-to-embryo transition. bioRxiv Processing (2021).

23 Liu, Y. et al. Conservation and divergence of poly(A) tail regulation during the mammalian oocyteto-embryo transition. bioRxiv Processing (2021).

24 Liu, Y., Nie, H., Liu, H. \& Lu, F. Poly(A) inclusive RNA isoform sequencing (PAlso-seq) reveals widespread non-adenosine residues within RNA poly(A) tails. Nat Commun 10, 5292, doi:10.1038/s41467-019-13228-9 (2019).

25 Reyes, J. M. \& Ross, P. J. Cytoplasmic polyadenylation in mammalian oocyte maturation. Wiley Interdiscip Rev RNA 7, 71-89, doi:10.1002/wrna.1316 (2016).

26 Schultz, R. M., Stein, P. \& Svoboda, P. The oocyte-to-embryo transition in mouse: past, present, and future. Biol Reprod 99, 160-174, doi:10.1093/biolre/ioy013 (2018).

27 Sanfins, A., Rodrigues, P. \& Albertini, D. F. GDF-9 and BMP-15 direct the follicle symphony. J Assist Reprod Genet 35, 1741-1750, doi:10.1007/s10815-018-1268-4 (2018).

28 Hikabe, O. et al. Reconstitution in vitro of the entire cycle of the mouse female germ line. Nature 539, 299-303, doi:10.1038/nature20104 (2016).

Garneau, N. L., Wilusz, J. \& Wilusz, C. J. The highways and byways of mRNA decay. Nat Rev Mol Cell Biol 8, 113-126, doi:10.1038/nrm2104 (2007).

Ma, J., Fukuda, Y. \& Schultz, R. M. Mobilization of Dormant Cnot7 mRNA Promotes Deadenylation of Maternal Transcripts During Mouse Oocyte Maturation. Biol Reprod 93, 48, doi:10.1095/biolreprod.115.130344 (2015).

31 Sha, Q. Q. et al. CNOT6L couples the selective degradation of maternal transcripts to meiotic cell 
cycle progression in mouse oocyte. EMBO J 37, doi:10.15252/embj.201899333 (2018).

$32 \mathrm{Lim}$, J. et al. Mixed tailing by TENT4A and TENT4B shields mRNA from rapid deadenylation. Science 361, 701-704, doi:10.1126/science.aam5794 (2018).

33 Hamazaki, N. et al. Reconstitution of the oocyte transcriptional network with transcription factors. Nature 589, 264-269, doi:10.1038/s41586-020-3027-9 (2021).

34 Yoshino, T. et al. Generation of ovarian follicles from mouse pluripotent stem cells. Science 373, eabe0237, doi:10.1126/science.abe0237 (2021).

35 White, Y. A. et al. Oocyte formation by mitotically active germ cells purified from ovaries of reproductive-age women. Nat Med 18, 413-421, doi:10.1038/nm.2669 (2012).

36 Hubner, K. et al. Derivation of oocytes from mouse embryonic stem cells. Science 300, 1251-1256, doi:10.1126/science.1083452 (2003). 


\section{Figure legends}

Fig. 1 | Abnormal maternal gene expression in mouse and human oocytes matured in vitro.

a, f, Illustration of the in vitro and in vivo oocyte maturation experiments in mouse (a) and human (f) oocytes.

$\mathbf{b}, \mathbf{g}$, Box plots for the normalized counts (in $\log 2$ scale) of genes $(\mathbf{b}, \mathrm{n}=14,131 ; \mathbf{g}, \mathrm{n}=$ $12,013)$ in germ-vesicle (GV) and in vivo maturated metaphase II (in vivo MII) or in vitro maturated metaphase II (in vitro MII) oocytes in mice (b) or humans (g).

$\mathbf{c}, \mathbf{h}$, Scatter plot for the normalized counts of individual genes in MII oocytes matured in vivo or in vitro in mice (c) or humans (h). Each dot represents one gene. Genes with at least $1 \mathrm{read}$ in one of the samples are included in the analysis. The number of genes included in the analysis and the numbers of differentially expressed genes are shown on the graphs. Genes upregulated in the in vitro MII oocytes are in red (c) or orange (h), while those downregulated in the in vitro MII oocytes are in blue. The differential expression is defined by a 2-fold cutoff.

d, i, Gene Ontology (GO) analysis of genes upregulated (red in $\mathbf{d}$ and orange in $\mathbf{i}$ ) or downregulated (blue) in the in vitro MII oocytes compared to in vivo MII oocytes in mice (d) or humans (i).

$\mathbf{e}, \mathbf{j}$, Normalized counts of Bmp15 in the in vitro and in vivo MII oocytes in mice (e) or humans (j). Error bars indicate the standard error of the mean (SEM) from two replicates. All $p$-values are calculated by Student's $t$-test. For all the box plots, the " $x$ " indicates the mean value, the black horizontal bars show the median value, and the top and bottom of the box represent the value of $25^{\text {th }}$ and $75^{\text {th }}$ percentile, respectively. The read counts are normalized by the counts of reads mapped to protein-coding genes in the mitochondria genome, if normalization is indicated. 
Fig. 2 mRNA deadenylation is impaired in mouse and human oocytes matured in vitro.

Histogram of poly(A) tails length of all transcripts (a, e), transcripts of the in vitro MII oocyte-upregulated genes $(\mathbf{b}$, red genes $(n=4,789)$ in Fig. $1 \mathbf{c} ; \mathbf{f}$, orange genes $(n=3,072)$ in Fig. 1h), transcripts of the in vitro MII oocyte-downregulated genes (c, blue genes ( $\mathrm{n}=$ 343) in Fig. 1c; $\mathbf{g}$, blue genes $(\mathrm{n}=1,352)$ in Fig. 1h), or transcripts of Bmp15 in in vivo or in vitro MII oocytes in mice $(\mathbf{a}-\mathbf{d})$ or humans $(\mathbf{e}-\mathbf{h})$. Histograms (bin size $=1 \mathrm{nt}$ ) are normalized by counts of reads mapped to protein-coding genes in the mitochondria genome. Transcripts with poly(A) tails of at least $1 \mathrm{nt}$ are included in the analysis. Transcripts with poly(A) tail lengths greater than $250 \mathrm{nt}(150 \mathrm{nt}$ for $\mathbf{d}$ and $200 \mathrm{nt}$ for $\mathbf{h})$ are included in the $250 \mathrm{nt}$ (150 nt for $\mathbf{d}$ and $200 \mathrm{nt}$ for $\mathbf{h})$ bin. 


\section{Fig. 3 | Mouse and human oocytes matured in vitro result in longer $\mathbf{N}$.}

a \& c, Diagram depicting mRNA with internal non-A residues in mice (a) and humans (c). $\mathrm{N}$ represents the length of residues between the end of 3' UTR and the first base of the longest consecutive $\mathrm{U}, \mathrm{C}$, or $\mathrm{G}$ residues in a poly(A) tail.

b, Histogram of the length of $\mathrm{N}$ and the ratio of $\mathrm{U} 1, \mathrm{U} 2, \mathrm{U} \geq 3, \mathrm{C}$, and $\mathrm{G}$ residues (from top to the bottom) in in vivo or in vitro MII oocytes in mice.

d, Histogram of the length of $\mathrm{N}$ and the ratio of $\mathrm{U} 1, \mathrm{U} 2-5, \mathrm{U} \geq 6, \mathrm{C}$, and $\mathrm{G}$ residues (from top to the bottom) in in vivo or in vitro MII oocytes in humans.

Histograms (bin size $=1 \mathrm{nt})$ are normalized to the total number of transcripts with a poly $(\mathrm{A})$ tail of at least $1 \mathrm{nt}$. Magnified views of the regions in the purple dotted squares are shown on the right. Red arrows highlight the proportion of transcripts with longer $\mathrm{N}$ increases in in vitro MII oocytes. 


\section{Fig. 4 | Polyadenylation of mRNAs encoding components of deadenylase complex is} impaired in mouse and human oocytes matured in vitro.

a, d, Normalized counts of Btg4 (left) and the four Cnot family (right) genes (Cnot6, Cnot6l, Cnot7, Cnot8) in the in vitro and in vivo MII oocytes in mice (a) and humans (d). Error bars indicate the SEM from two replicates $(n=2)$. Fold changes for each gene are shown on top of the column.

b, e, Box plot for the poly(A) tail length of Btg4 and the four Cnot family genes in GV and MII oocytes matured in vivo or in vitro in mice (b) and humans (e). For all the box plots, the " $x$ " indicates the mean value, the horizontal bars show the median value, and the top and bottom of the box represent the value of $25^{\text {th }}$ and $75^{\text {th }}$ percentile, respectively.

c, Box plot of translational efficiency $(\mathrm{TE}, \text { in } \log 2 \text { scale })^{31}$ of genes $(\mathrm{n}=2,951)$ divided by the length of poly(A) tails in mouse in vivo MII oocytes. The $p$-values tested by Student's $t$-test between each of the two groups are shown on the top right. Examples of poly(A) tail length and TE for Btg4, Tcl1b4, Wee2, Suz12, Tgfb2, and Atg5 are shown on the bottom right. 

maturation in vitro permission.

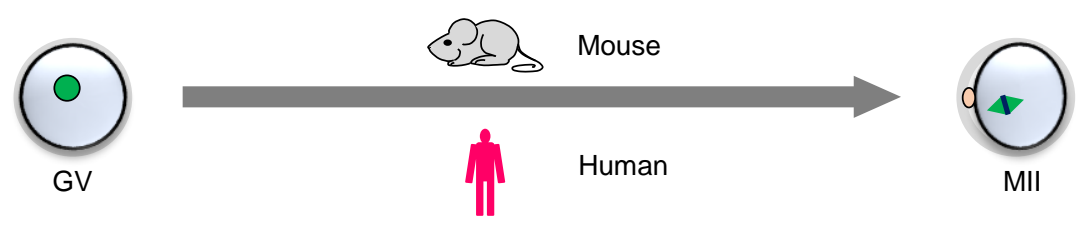

mRNAs for most genes:

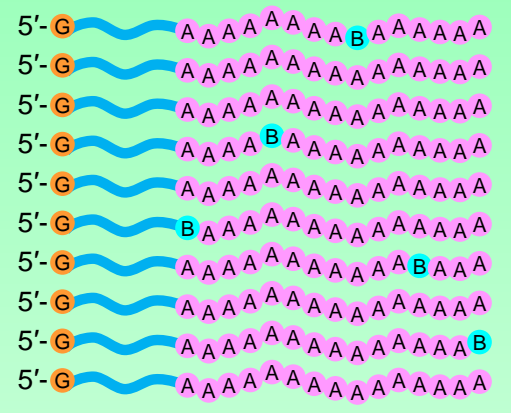

Btg4 mRNAs:

5'- $={ }_{A A B A}$

Cnot6/6I/7/8 mRNAs:

$5^{\prime}-\mathrm{G}=\mathrm{AAA}_{A} A^{A}$

No or low translation

Poly $(\mathrm{A})$ tail dynamics during maturation in vivo

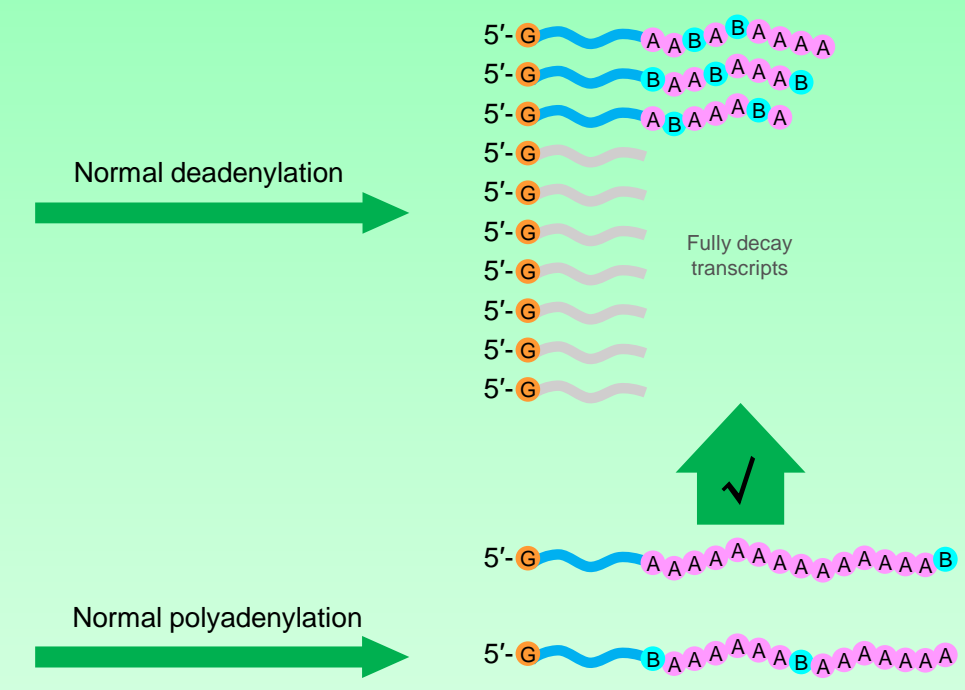

High translation

Poly $(\mathrm{A})$ tail dynamics during maturation in vitro

mRNAs for most genes:

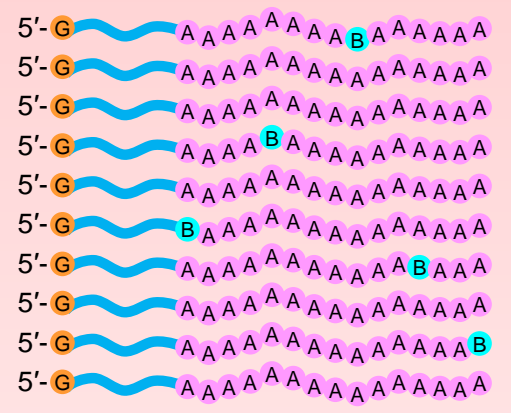

Btg4 mRNAs:

$$
5^{\prime}-\mathrm{G}=\mathrm{AABA}^{\mathrm{A}}
$$

Cnot6/61/7/8 mRNAs:

$$
5^{\prime}-\mathrm{G}=\mathrm{AAAA}^{\mathrm{A}}
$$

$\checkmark$ No or low translation

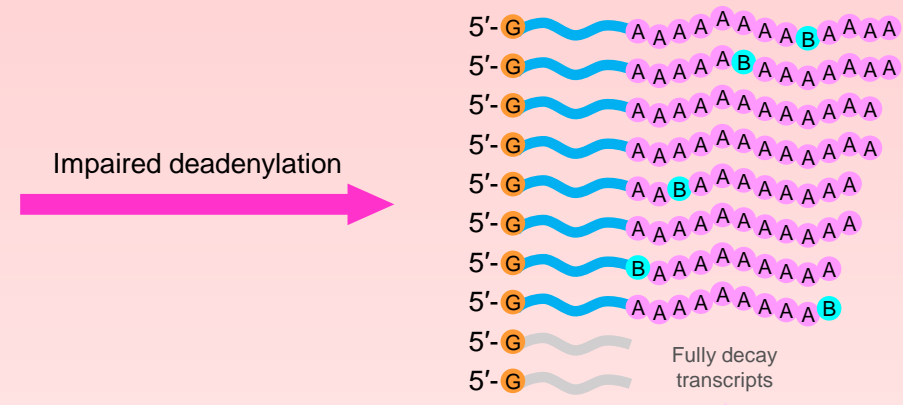

\section{$x$}

$5^{\prime}-\mathrm{G}=\mathrm{A}_{\mathrm{A} A} A^{A} \mathrm{~A}_{\mathrm{A} A}$

Impaired polyadenylation

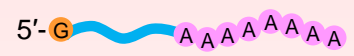

No or low translation 


\section{Fig. 5 | Summary of impaired maternal mRNA deadenylation during oocyte} maturation in vitro.

During oocyte maturation in vivo (up), the polyadenylation of mRNAs encoding components of deadenylase complex is normal, which results in high deadenylase abundance by high translation efficiency. Therefore, the global maternal mRNA deadenylation is normal.

During oocyte maturation in vitro (down), the polyadenylation of mRNAs encoding components of deadenylase complex is impeded, which results in low deadenylase abundance by low translation efficiency. Therefore, the global maternal mRNA deadenylation is impaired. 


\section{Extended Data Fig. 1 | Proportion of transcripts containing non-A residues in the in vitro and in vivo MII oocytes in mice and humans.}

a, e, Overall proportion of poly(A) tails containing $\mathrm{U}, \mathrm{C}$, or $\mathrm{G}$ residues in the in vitro and in vivo MII oocytes in mice (a) and humans (e). Error bars indicate the SEM from two replicates and the $p$-value is calculated by Student's $t$-test (a).

b, Diagram depicting mRNA with 5'-end, internal, and 3'-end non-A residues.

c, f, Proportion of poly(A) tails containing U, C, or G residues in 5'-end and internal in the in vitro and in vivo MII oocytes in mice (c) and humans (f). The U residues were further divided according to the length of the longest consecutive $U(1,2$, and $\geq 3)$.

d, $\mathbf{g}$, Box plot for the proportion of reads containing $U, C$, or $\mathrm{G}$ residues of individual genes in the in vitro and in vivo MII oocytes in mice (d) and humans (g). Transcripts with poly(A) tails of at least $1 \mathrm{nt}$ for a gene with at least 20 reads $(\mathbf{d}, \mathrm{n}=5,845 ; \mathbf{g}, \mathrm{n}=3,163,2,745$, and 2,762 for $\mathrm{U}, \mathrm{C}$, and $\mathrm{G}$, respectively) are included in the analysis. The $p$-value is calculated by Student's $t$-test. For all the box plots, the " $x$ " indicates the mean value, the black horizontal bars show the median value, and the top and bottom of the box represent the value of $25^{\text {th }}$ and $75^{\text {th }}$ percentile, respectively. 


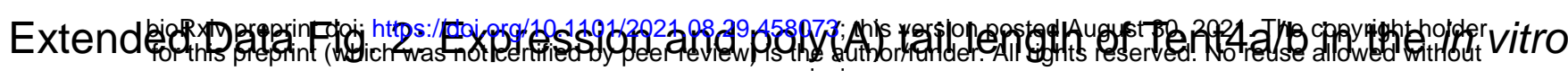

and in vivo Mll oocytes in mice and hurpertission.

\section{Co Mouse}

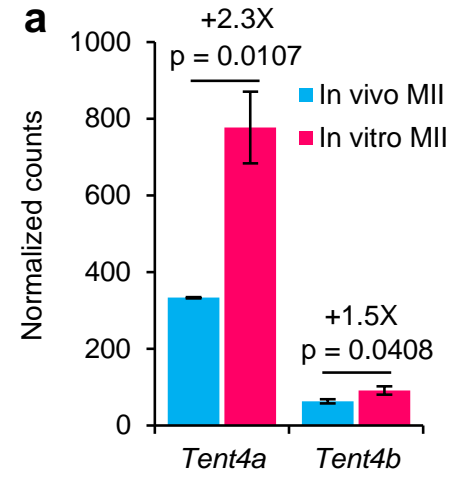

b

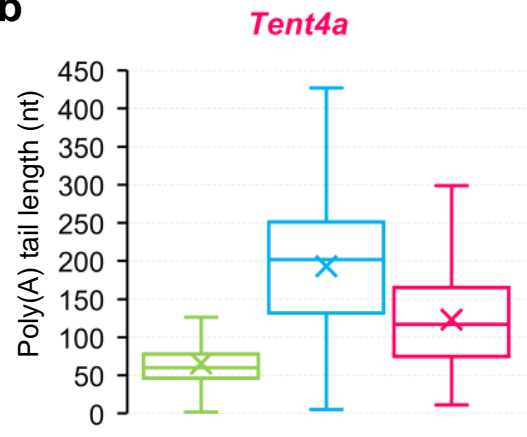

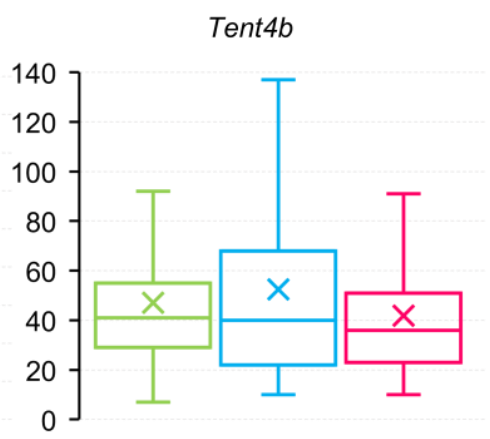

GV

$\square$ In vivo MII

$\square$ In vitro MII
Human

C

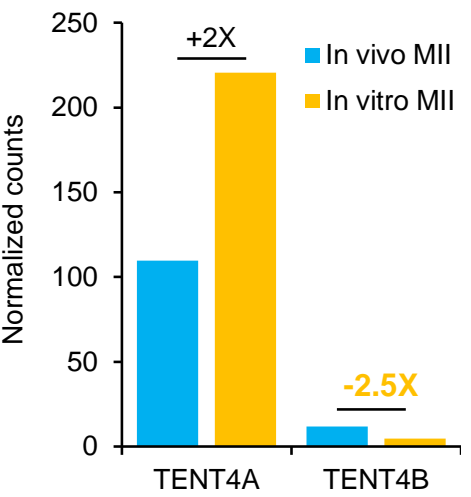

d

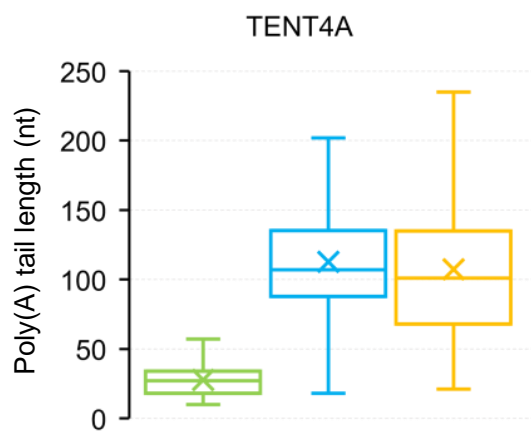

TENT4B

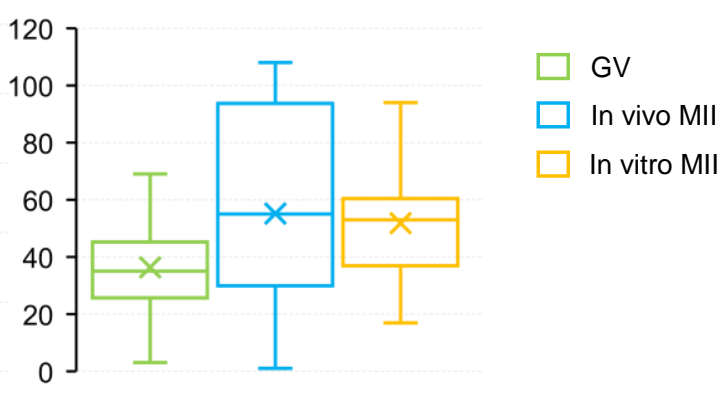


Extended Data Fig. 2 | Expression and poly(A) tail length of Tent4a/b in the in vitro and in vivo MII oocytes in mice and humans.

a, c, Normalized counts of Tent4a and Tent $4 b$ in the in vitro and in vivo MII oocytes in mice (a) and humans (c). Error bars indicate the SEM from two replicates and the $p$-value is calculated by Student's $t$-test (a).

b, d, Box plot for the poly(A) tail length of Tent4a and Tent4b in GV and MII oocytes matured in vivo or in vitro in mice (b) and humans (d). For all the box plots, the " $\times$ " indicates the mean value, the horizontal bars show the median value, and the top and bottom of the box represent the value of $25^{\text {th }}$ and $75^{\text {th }}$ percentile, respectively. 